\title{
Características tecnológicas, sensoriais e químicas de massas secas sem glúten à base de farinhas de sorgo e milho
}

\section{Technological, sensory and chemical characteristics of gluten-free pasta made from sorghum and corn flours}

Caroline Liboreiro Paiva ${ }^{{ }^{*}}$ (1), Valéria Aparecida Vieira Queiroz², Maria Aparecida Vieira Teixeira Garcia ${ }^{3}$

\author{
${ }^{1}$ Universidade Federal de Minas Gerais (UFMG), Instituto de Ciências Agrárias, Montes Claros/MG - Brasil \\ ${ }^{2}$ Embrapa Milho e Sorgo, Sete Lagoas/MG - Brasil \\ 3Universidade Federal de Minas Gerais (UFMG), Faculdade de Farmácia, Belo Horizonte/ MG - Brasil
}

${ }^{*}$ Corresponding Author: Caroline Liboreiro Paiva, Universidade Federal de Minas Gerais (UFMG), Instituto de Ciências Agrárias, Av. Universitária, 1000, CEP: 39404-5470, Montes Claros/MG - Brasil, e-mail: carolinepaiva7@gmail.com

Cite as: Paiva, C. L., Queiroz, V. A. V., \& Garcia, M. A. V. T. Technological, sensory and chemical characteristics of gluten-free pasta made from sorghum and corn flours. Brazilian Journal of Food Technology, 22, e2018095. https://doi.org/10.1590/1981-6723.09518

\begin{abstract}
Resumo
O único tratamento para a doença celíaca é uma dieta isenta de glúten por toda a vida, o que torna necessária a oferta de novos produtos para esse público. O sorgo e o milho são cereais que, além de apresentarem propriedades funcionais, são possíveis de serem inseridos como ingredientes em dietas sem glúten, por não possuírem, em sua composição, as proteínas formadoras de glúten. Assim, este estudo objetivou elaborar massas secas utilizando farinha de sorgo e de milho, e avaliar algumas características químicas e de cozimento, assim como a aceitabilidade sensorial dos produtos formulados, frente a um painel de indivíduos celíacos e não celíacos. As amostras incluíram uma massa elaborada unicamente com farinha de sorgo, outra com $50 \%$ de farinha de sorgo e $50 \%$ de farinha de milho, e uma terceira, à base unicamente de farinha de milho. O produto elaborado com $100 \%$ de farinha de sorgo apresentou conteúdos significativamente mais altos de proteína, lipídeos, cinzas, fibra e fenólicos totais, em comparação com os demais. A perda de sólidos dos produtos variou de 5,04\% a 10,54\%, parâmetro de qualidade considerado adequado para macarrão. Após cozimento, as três massas absorveram mais de duas vezes os seus pesos em água (108,1 a 143,1\%). Embora o público composto por pessoas não celíacas tenha preferido a massa à base de farinha de milho, aquela elaborada unicamente com farinha de sorgo apresentou aceitabilidade satisfatória entre os provadores portadores da doença celíaca, o que demonstra o potencial deste ingrediente em macarrões destinados a este público.
\end{abstract}

Palavras-chave: Sorghum bicolor (L) Moech; Zea mays; Macarrão sem glúten; Qualidade de cozimento; Composição química; Teste de aceitação. 


\begin{abstract}
The only treatment for celiac disease is a gluten free diet for the whole life of the person and hence new products must be offered to this public. Sorghum and corn are cereals that, besides having functional properties, do not contain gluten-forming proteins in their composition and can therefore be inserted as ingredients into gluten-free diets. The objectives of this study were to prepare dry pasta using maize and sorghum flours and to evaluate some of the chemical and cooking characteristics, as well as the sensory acceptability of the formulated products according to a panel of celiac and non-celiac individuals. The samples included one made with only sorghum flour, another with $50 \%$ sorghum flour and $50 \%$ corn flour, and a third made with only corn flour. The product elaborated with $100 \%$ of sorghum flour had significantly higher total protein, lipid, ash, fibre and total phenolic compound contents then the others. The loss of solids of the products ranged from $5.04 \%$ to $10.54 \%$, a quality parameter considered adequate for pasta. After cooking, the three pastas absorbed more than twice their weights in water (108.1 to $143.1 \%$ ). Although the public made up of non-celiac individuals preferred the pasta made with only corn flour, the pasta made with only sorghum flour showed satisfactory acceptability amongst testers with celiac disease, which demonstrates the potential of this ingredient in pasta intended for this public.
\end{abstract}

Keywords: Sorghum bicolor (L) Moech; Zea mays; Gluten-free pasta; Cooking quality; Chemical composition; Acceptability test.

\title{
1 Introdução
}

A doença celíaca (DC) é uma desordem autoimune causada pela ingestão de proteínas formadoras de glúten em indivíduos geneticamente susceptíveis, mais especificamente sensíveis à prolamina, presente principalmente em trigo, cevada e centeio. A atrofia das vilosidades intestinais é uma das manifestações iniciais da doença, o que resulta em baixa absorção de macro e micronutrientes (Araújo et al., 2010).

$\mathrm{O}$ tratamento da doença consiste em uma dieta totalmente isenta de glúten, o que restringe a alimentação desses indivíduos, visto que, especialmente no mercado brasileiro, a oferta de alimentos sem glúten, embora em expansão, ainda é restrita, pois a maioria das massas, incluindo produtos de panificação, além de pizzas e macarrões, é formulada com farinha de trigo. Segundo a ACELBRA (Associação dos Celíacos do Brasil, 2017), em pesquisa realizada junto aos portadores de doença celíaca, depois dos pães, o macarrão é o segundo produto que esse público gostaria de encontrar no mercado de produtos alimentícios. Os celíacos também relatam que a oferta de alimentos sensorialmente apropriados é restrita, o que torna a dieta monótona, e que os produtos disponíveis no mercado são normalmente de alto custo (Araújo et al., 2010). Assim, é necessário desenvolver produtos sem glúten para esse mercado e estudar as características sensoriais demandadas. Vale destacar que poucos trabalhos têm avaliado os atributos sensoriais requeridos pelos indivíduos celíacos.

O sorgo é um cereal com potencial para ser empregado como ingrediente em alimentos sem glúten, por ser isento de proteínas formadoras de glúten e por possuir sabor neutro, o que amplia as possibilidades de sua inserção em diversas formulações. Esse cereal também é fonte de nutrientes, especialmente de carboidratos, fibras (Queiroz et al., 2015), amido resistente (Dicko et al., 2006; Teixeira et al., 2016) e de minerais, como zinco (Paiva et al., 2017). Também é rico em compostos fenólicos, como poliflavanóis, antocianinas e ácidos fenólicos (Awika \& Rooney, 2004). Vários trabalhos têm demonstrado os efeitos benéficos do sorgo para a saúde humana, tais como redução de dislipidemias, inflamações, obesidade, câncer e doença cardiovascular (Arbex et al., 2018; Awika et al., 2004, 2005; Awika \& Rooney, 2004; Middleton Junior et al., 2000; Moraes et al., 2012). Especialmente, Khan et al. (2015) demonstraram que macarrão contendo farinha integral de sorgo de pericarpo vermelho apresentou atividade antioxidante e capacidade de melhorar os marcadores de estresse oxidativo em indivíduos saudáveis submetidos à dieta com esse produto. Além disso, a cultura do sorgo é tolerante a condições ambientais secas e quentes, nas quais o cultivo de outros cereais é antieconômico. Geralmente, apresenta menor custo de produção, pelo fato de a cultura ser mais eficiente no 
uso da água e de nutrientes do solo, sendo o cereal comercializado por um valor mais baixo no mercado, em relação ao milho e ao arroz (Queiroz et al., 2012).

De acordo com Phimolsiripol et al. (2012), os produtos sem glúten geralmente são de baixa qualidade quando comparados aos produtos convencionais. Em geral, apresentam baixa qualidade sensorial e são pobres em proteínas e fibras alimentares. Portanto, o uso de sorgo no desenvolvimento de produtos poderá contribuir para a melhoria da qualidade nutricional e sensorial de produtos destinados a esse público.

Devido a sua facilidade de produção e versatilidade, o sorgo tem sido utilizado em outros países como base alimentar de milhares de pessoas. Todavia, no mercado brasileiro, a inserção do sorgo na alimentação humana é ainda recente. Alguns produtos à base de sorgo já foram propostos em trabalhos acadêmicos, como barras de cereais (Paiva et al., 2018; Queiroz et al., 2012), cookies (Ferreira et al., 2009), preparados em pó para bebida de baixa caloria (Queiroz et al., 2018) e cereais matinais (Anunciação et al., 2017), todos com as propostas de diversificar receitas e oferecer alternativas de consumo a pessoas intolerantes ao glúten.

Assim, este estudo teve como objetivos elaborar massas secas utilizando farinha de sorgo e de milho, e avaliar algumas características químicas e de cozimento, assim como a aceitabilidade sensorial dos produtos formulados, frente a um painel de indivíduos celíacos e não celíacos.

\section{Material e métodos}

As amostras avaliadas incluíram três produtos, elaborados com: (1) $100 \%$ de farinha de sorgo (MS); (2) $50 \%$ de farinha de sorgo e $50 \%$ de farinha de milho (MSM), e (3) $100 \%$ de farinha de milho (MM), sendo este último um produto comercial, usado como controle. Os produtos foram elaborados a partir de grãos integrais e fabricados pela TIVVA® (Sorocaba, São Paulo), no formato espiralado (Fusilli). A farinha de sorgo empregada nas formulações foi proveniente da cultivar BRS506, grão de pericarpo cinza, desenvolvido e cultivado nos campos experimentais da Embrapa Milho e Sorgo (Sete Lagoas, Minas Gerais) $\left(19^{\circ} 28^{\prime} \mathrm{S}\right.$ e $44^{\circ} 15^{\prime} \mathrm{W}$ ), no período de março a julho de 2015 , sob condições controladas.

As massas secas foram moídas até obter partículas de tamanho médio de 0,5 mm (Marconi, MA048, Piracicaba, São Paulo), empacotadas em sacos de polietileno e, em seguida, armazenadas sob congelamento até a realização das análises químicas, todas realizadas em triplicata.

As fibras em detergente neutro foram determinadas usando $0,5 \mathrm{~g}$ de amostra em um analisador de fibra (Tecnal, EQ LCC 08, Piracicaba, São Paulo), sistema Ankom com sacos filtrantes (ANKOM Technology, 2006). Os lipídeos foram determinados em $1 \mathrm{~g}$ de amostra, usando extrator de gordura (Ankom, XT10, Macedon, NY, EUA), seguindo o protocolo AOCS (American Oil Chemists' Society, 2004). O teor de proteína foi determinado pelo método de Dumas (Wiles et al., 1998) em $0,25 \mathrm{~g}$ de amostra, utilizando um analisador de nitrogênio (Leco, FP-528, Rio de Janeiro, Rio de Janeiro). Fator de conversão de 6,25 foi utilizado para expressar o teor de nitrogênio total em proteína. Cinzas foram determinadas em $2 \mathrm{~g}$ de amostra, de acordo com método da AOAC (Association of Official Analytical Chemists, 2016), com calcinação da matéria orgânica em mufla (Quimis, Q318A24, Diadema, São Paulo) a $600{ }^{\circ} \mathrm{C}$ durante $2 \mathrm{~h}$. A concentração de carboidratos foi determinada por diferença entre a matéria seca e os conteúdos de proteínas, lipídeos, fibras e cinzas.

Os resultados foram expressos em base seca, sendo a matéria seca determinada pelo método gravimétrico em $2 \mathrm{~g}$ de amostra, usando estufa com circulação forçada de ar a $105^{\circ} \mathrm{C}$ por $6 \mathrm{~h}$.

A extração dos compostos fenólicos foi conduzida de acordo com a metodologia de Dykes et al. (2005). Amostras de $250 \mathrm{mg}$ foram extraídas com $25 \mathrm{~mL}$ de metanol acidificado $(1 \% \mathrm{HCl}, \mathrm{v} / \mathrm{v})$, por $2 \mathrm{~h}$, em mesa agitadora a $150 \mathrm{rpm}$ e, em seguida, centrifugadas a $3.000 \mathrm{rpm}$ por $15 \mathrm{~min}$. Foram adicionados, em um tubo de centrífuga, $100 \mu \mathrm{L}$ do sobrenadante, $1,1 \mathrm{~mL}$ de água, $0,4 \mathrm{~mL}$ do reagente Folin-Ciocalteu $(0,02 \mathrm{~N})$ e $0,9 \mathrm{~mL}$ de etanolamina $(0,5 \mathrm{M})$. Após agitação em vortex, seguido de 20 min de repouso para reação, a absorbância foi medida em espectrofotômetro a $600 \mathrm{~nm}$, à temperatura ambiente. A análise foi feita em triplicata. 
Soluções de ácido gálico nas concentrações de 50,100, 150 e $200 \mathrm{mg} / \mathrm{L}$ foram preparadas e usadas para construção da curva padrão $\left(\mathrm{y}=0,0062+0,0038 \mathrm{x} ; \mathrm{R}^{2}=0,9974\right)$. Os níveis de fenólicos totais foram expressos em miligrama equivalente de ácido gálico por grama de amostra.

Parâmetros de cozimento, como tempo de cozimento, ganho de peso, perda de sólidos e firmeza, foram determinados segundo o método 66-50 (AACC International Approved Methods, 2010), após o cozimento das massas.

O tempo de cozimento foi determinado após a inserção de $10 \mathrm{~g}$ de amostra em um Béquer contendo $150 \mathrm{~mL}$ de água destilada em ebulição. Esse parâmetro foi determinado removendo-se pedaços da massa da água de cozimento em intervalos de $30 \mathrm{~s} \mathrm{e}$, em seguida, espremendo estes entre duas placas de vidro. O tempo de cozimento foi estabelecido quando não mais se observou o hilo branco central no pedaço de massa.

O ganho de peso foi determinado pela pesagem de $10 \mathrm{~g}$ de amostra antes e depois do cozimento em $150 \mathrm{~mL}$ de água destilada, de acordo com o tempo de cozimento de cada amostra. Quando o tempo de cozimento foi atingido, a amostra foi drenada em funil de Büchner e rinsada com $30 \mathrm{~mL}$ de água destilada. Esse valor foi expresso em porcentagem.

A água de cozimento e a água utilizada para rinsagem foram combinadas para a determinação da perda de sólidos após cozimento. Uma alíquota de $25 \mathrm{~mL}$ da água de cozimento foi coletada em um recipiente e colocada em estufa a $105^{\circ} \mathrm{C}$ até completa secagem. O resíduo foi pesado e a porcentagem de perda de sólidos foi calculada de acordo com a Fórmula 1:

Perda de sólidos $(\%)=\frac{[\text { peso do resíduo }(\mathrm{g}) \times \text { volume da água de cozimento e de rinsagem }(\mathrm{mL}) \times 100]}{[\text { Peso da amostra }(\mathrm{g}) \times 25(\mathrm{~mL})]}$

Para a avaliação sensorial, as amostras de macarrão foram cozidas em solução salina (4\%), na proporção de $100 \mathrm{~g}$ de massa por litro de solução. O macarrão foi servido à temperatura aproximada de $50{ }^{\circ} \mathrm{C}$ com molho de tomate, forma típica de consumo no Brasil. Um painel não treinado de 74 provadores (55 mulheres e 19 homens, entre 20 e 44 anos), entre estudantes de graduação e funcionários da Universidade Federal de São João Del Rei (UFSJ), campus Sete Lagoas, Minas Gerais, e 39 indivíduos celíacos (33 mulheres e seis homens, acima de 18 anos), associados à ACELBRA/MG, fizeram a avaliação sensorial dos macarrões, usando escala hedônica 10 híbrida $(0=$ desgostei extremamente; $10=$ gostei extremamente $)$ (Villanueva et al., 2005). Na UFSJ, cada provador experimentou as três amostras e, na ACELBRA, cada indivíduo celíaco experimentou apenas o macarrão de sorgo, porque havia garantia de ausência de vestígios de glúten apenas no macarrão à base de farinha de sorgo. Somente os grãos de sorgo foram cultivados e moídos na Embrapa, sob condições controladas. O teste sensorial foi conduzido em cabines individuais, com iluminação controlada (branca). Os provadores receberam aleatoriamente $20 \mathrm{~g}$ de amostras em potes plásticos codificados com números aleatórios de três dígitos. As amostras foram servidas juntamente com um copo de água mineral à temperatura ambiente. As amostras foram distribuídas de forma monádica, aleatória, com delineamento em blocos completos balanceados.

O Comitê de Ética em Pesquisa da Universidade Federal de Minas Gerais (UFMG), Brasil, aprovou este estudo sob o código 03591312.0.0000.5149.

Os resultados da análise da composição química, de fenólicos totais, dos parâmetros de cozimento e da análise sensorial dos três tipos de macarrão foram submetidos à análise de variância (ANOVA) e as médias foram comparadas pelo Teste de Tukey a 5\% de probabilidade, usando o programa SISVAR, versão 5.6 (UFLA, Lavras, Minas Gerais). 


\section{Resultados e discussão}

A composição química dos macarrões está descrita na Tabela 1. A amostra MS apresentou composição significativamente mais alta $(p<0,05)$ de proteína, lipídeos, fibra e cinzas, em relação aos demais produtos. Ao contrário, o MS apresentou conteúdo significativamente mais baixo de carboidratos.

Tabela 1. Composição química* dos macarrões.

\begin{tabular}{ccccccc}
\hline \multirow{2}{*}{ Amostras } & \multicolumn{7}{c}{ Média $( \pm$ desvio padrão) } \\
\cline { 2 - 7 } & $\begin{array}{c}\text { Proteína } \\
(\mathbf{g} / \mathbf{1 0 0 g})\end{array}$ & $\begin{array}{c}\text { Carboidratos } \\
\mathbf{( g / 1 0 0 g})\end{array}$ & $\begin{array}{c}\text { Lipídeos } \\
\mathbf{( g / 1 0 0 g})\end{array}$ & $\begin{array}{c}\text { Fibra } \\
(\mathbf{g} / \mathbf{1 0 0 g})\end{array}$ & $\begin{array}{c}\text { Cinzas } \\
(\mathbf{g} / \mathbf{1 0 0 g})\end{array}$ & $\begin{array}{c}\text { Fenólicos totais } \\
(\mathbf{m g} \text { EAG/g) }\end{array}$ \\
\hline MS & $12,34 \pm 0,14^{\mathrm{a}}$ & $66,86 \pm 0,30^{\mathrm{c}}$ & $1,10 \pm 0,01^{\mathrm{a}}$ & $8,20 \pm 0,12^{\mathrm{a}}$ & $1,63 \pm 0,01^{\mathrm{a}}$ & $23,67 \pm 0,84^{\mathrm{a}}$ \\
MSM & $9,57 \pm 0,04^{\mathrm{b}}$ & $73,55 \pm 0,44^{\mathrm{b}}$ & $0,60 \pm 0,06^{\mathrm{b}}$ & $4,72 \pm 0,34^{\mathrm{b}}$ & $1,41 \pm 0,06^{\mathrm{b}}$ & $18,29 \pm 0,99^{\mathrm{b}}$ \\
\hline MM & $6,22 \pm 0,03^{\mathrm{c}}$ & $80,31 \pm 0,18^{\mathrm{a}}$ & $0,70 \pm 0,02^{\mathrm{b}}$ & $2,08 \pm 0,11^{\mathrm{c}}$ & $0,72 \pm 0,02^{\mathrm{c}}$ & $7,92 \pm 0,32^{\mathrm{c}}$ \\
\hline
\end{tabular}

*Média de 3 repetições. Valores em massa seca. Médias seguidas de letras diferentes na mesma coluna são significativamente diferentes (Teste de Tukey, $p<0,05$ ). MS: macarrão elaborado com $100 \%$ de farinha de sorgo; MSM: macarrão elaborado com $50 \%$ de farinha de sorgo e $50 \%$ de farinha de milho; MM: macarrão elaborado com $100 \%$ de farinha de milho.

Ferreira et al. (2016) detectaram conteúdos mais baixos de cinzas $(0,75$ a 0,87\%) e proteína $(7,86$ a 8,85\%) em massas secas elaboradas com diferentes proporções de farinhas de sorgo, milho, arroz e batata. Ao contrário, detectaram maiores teores de lipídeos (4,41 a 4,86\%), provavelmente pelo fato de essas formulações conterem também óleo e ovos. Giuberti et al. (2015) formularam macarrão com $100 \%$ de farinha de arroz e encontraram menores teores de proteína $(9,0 \%)$ e fibra dietética $(1,2 \%)$, em relação às massas MS e MSM. Também os teores de cinzas foram menores que todas as massas (MS, MSM e MM). Já o teor de carboidratos $(82,6 \%)$ foi maior e o de lipídeos $(1,1 \%)$, similar, comparados ao presente estudo. Tomicki et al. (2015) elaboraram macarrões com diversas proporções de farinha de arroz e milho. Os resultados de proteínas desses produtos variaram de 6,8 a 10,3\%, portanto mais baixos que a massa MS elaborada em nosso estudo. Também, os valores encontrados para carboidratos $(49,1$ a $53,8 \%)$ foram mais baixos que os determinados em todas as massas deste estudo. No entanto, os valores de lipídeos foram maiores (1,3 a 3,9\%), provavelmente pela adição de ovos. A massa elaborada por Petitot et al. (2010), unicamente com farinha de trigo durum, apresentou valores ligeiramente mais altos para alguns componentes nutricionais $(13,3 \%$ de proteína, $1,7 \%$ de lipídeos e 77,6\% de carboidratos), em comparação ao MS. Já os valores de fibra (2,4\%) e cinzas foram mais baixos do que os do MS.

Em relação aos fenólicos totais, a massa elaborada unicamente com farinha de sorgo apresentou valor significativamente mais alto que os demais produtos (Tabela 1). Da mesma forma, o macarrão elaborado com sorgo e milho apresentou teor significativamente mais alto que o feito apenas com milho. Sabe-se que os compostos fenólicos do grão de sorgo estão localizados principalmente no pericarpo. Assim, o emprego da farinha integral de sorgo nos macarrões justifica esse resultado. Sęczyk et al. (2016) avaliaram o conteúdo de fenólicos totais em macarrão à base de semolina de trigo durum e encontram valores bem menores (3,51 mg EAG/g), em comparação aos produtos desenvolvidos neste trabalho. Vale destacar ainda que, geralmente, sorgos de pericarpo preto, bronze e vermelho possuem quantidades maiores de fenólicos, devido ao conteúdo de antocianinas (Awika \& Rooney, 2004). O sorgo empregado na elaboração das massas foi de pericarpo claro, cinza, sendo provável que outros compostos fenólicos, como flavonas, flavanonas e ácidos fenólicos, tenham contribuído para o resultado de fenólicos totais.

A Tabela 2 apresenta os resultados dos parâmetros de cozimento das massas preparadas com farinhas de sorgo e de milho. O tempo de cozimento das massas sem glúten variou de 5,5 a 7,5 min. Em geral, os tempos de cozimento refletem a cristalinidade do amido ou a sua resistência à gelatinização. Esses resultados sugerem que o amido de milho tem uma estrutura mais organizada do que o amido de sorgo. 
Tabela 2. Parâmetros de qualidade* do cozimento dos macarrões à base de farinha de sorgo e de milho.

\begin{tabular}{|c|c|c|c|}
\hline \multirow[b]{2}{*}{ Amostras } & \multicolumn{3}{|c|}{ Média ( \pm desvio padrão) } \\
\hline & $\begin{array}{c}\text { Tempo de cozimento } \\
\text { ( } \mathrm{min})\end{array}$ & $\begin{array}{c}\text { Aumento de peso } \\
(\%)\end{array}$ & $\begin{array}{c}\text { Perda de sólidos } \\
(\%)\end{array}$ \\
\hline MS & 5,5 & $122,7 \pm 9,39^{\mathrm{b}}$ & $5,04 \pm 0,25^{b}$ \\
\hline MSM & 5,5 & $143,1 \pm 8,67^{\mathrm{a}}$ & $5,80 \pm 0,30^{b}$ \\
\hline MM & 7,5 & $108,1 \pm 5,25^{\mathrm{b}}$ & $10,54 \pm 0,48^{\mathrm{a}}$ \\
\hline
\end{tabular}

*Média de 3 repetições. Médias seguidas de letras diferentes na mesma coluna são significativamente diferentes (Teste de Tukey, $p \leq 0,05$ ). MS: macarrão elaborado com $100 \%$ de farinha de sorgo; MSM: macarrão elaborado com $50 \%$ de farinha de sorgo e $50 \%$ de farinha de milho; MM: macarrão elaborado com $100 \%$ de farinha de milho.

De acordo com Hummel (1966), um macarrão de boa qualidade deve absorver água durante o cozimento, pelo menos, numa proporção duas vezes maior que a sua massa inicial. Baseando-se nesse fato, verifica-se que as três massas apresentaram aumento de peso satisfatório (108,1 a 143,1\%), embora a amostra MSM tenha apresentado aumento de peso significativamente maior que as demais.

O aumento de peso ou a absorção de água está relacionado com o alto teor de polissacarídeos não amiláceos na formulação, mas depende também da conformação dos grânulos de amido, da presença de áreas mais amorfas e da ruptura da matriz proteica durante o cozimento (Alamprese et al., 2007). Além disso, depende das características geométricas da massa (Lucisano et al., 2012). Assim, na literatura, esse valor varia muito, mas comparando com o nosso estudo, observou-se um valor similar de ganho de peso para macarrão de arroz e macarrão de milho elaborados por Lucisano et al. (2012). As massas elaboradas com diferentes proporções de farinha de arroz e de sorgo apresentaram valores mais baixos: 70,7 a 75,7\% (Marengo et al., 2015). Do mesmo modo, o aumento de peso após o cozimento de espaguete feito de farinha de arroz e feijão foi menor: 70,0 a 109,3\% (Giuberti et al., 2015). No entanto, para massas feitas com uma mistura de farinha de arroz, sorgo e batata, o ganho de peso foi $260 \%$ (Ferreira et al., 2016). Espaguetes elaborados a partir de 16 cultivares de trigo durum tiveram aumento de peso após cozimento maiores que o resultado do presente estudo (205 a 227\%) (Abuhammad et al., 2012).

A perda de sólidos na água de cozimento dos produtos MS, MSM e MM variou de 5,04\% a 10,54\% (Tabela 2). As amostras contendo farinha de sorgo (MS e MSM) tiveram uma perda de sólidos significativamente menor durante o cozimento do que a massa feita apenas com farinha de milho (MM). De acordo com Hummel (1966), perda de sólidos menor que 6\% é um bom resultado; até $8 \%$ é normal, e 10\% ou mais é ruim, devido à grande exsudação de amido na água de cozimento. Normalmente, as pastas sem glúten mostram altos valores de perda de sólidos, devido à ausência de um retículo proteico bem estruturado, o que promove um inchaço excessivo dos grânulos de amido e sua consequente exsudação na água de cozimento (Alamprese et al., 2007; Petitot et al., 2010). Susanna \& Prabhasankar (2012) relataram valores de perda de sólidos variando de 5,66 a 7,42\% para massas feitas de trigo durum. Fusilli de trigo durum apresentou perda de sólidos de 4,5 e 5,0\% (Güler et al., 2002) e espaguete, 5,6\% (Petitot et al., 2010). Espaguete sem glúten elaborado com farinha de arroz apresentou perda de sólidos de 12,9\% (Giuberti et al., 2015) e elaborado com farinha de milho, 9\% (Lucisano et al., 2012). Macarrões cortados e com $7 \mathrm{~mm}$ de diâmetro, elaborados com farinha de arroz ou farinha de sorgo, apresentaram perda de sólidos de 11,5\% e $8,4 \%$, respectivamente (Marengo et al., 2015).

De acordo com Biliaderis (1992), durante o cozimento da massa, a estrutura cristalina do amido é quebrada devido ao relaxamento das ligações de hidrogênio e ao fato de as moléculas de água interagirem com os grupos hidroxila da amilose e amilopectina, causando aumento do tamanho de grânulos e dissolução parcial de amido. A associação da amilose e amilopectina no processo de retrogradação promove a firmeza da massa cozida, reduzindo a perda de sólidos. Assim, as propriedades de cozimento dependem do tipo de matériaprima, especialmente da fonte de amido e da presença ou ausência de proteína na formulação.

Quanto às avaliações sensoriais, os julgadores celíacos gostaram significativamente mais do macarrão de sorgo, em comparação com os julgadores não celíacos, em relação à qualidade global e aos atributos de cor, 
sabor e textura do produto (Figura 1). O painel de indivíduos não celíacos, que provou todos os macarrões, demonstrou preferência significativa pelos quesitos sabor, textura e qualidade global da amostra MM (Tabela 3). Os atributos de cor e textura do macarrão MSM foram mais aceitos do que os da amostra MS. No entanto, estes foram igualmente aceitos em relação ao sabor e à qualidade global. Ferreira et al. (2016) demonstraram que massas sem glúten têm menor capacidade de reter os grânulos de amido, o que reflete em textura mais pegajosa do produto. Essa textura pegajosa ou aderente poderia ser uma possível causa da baixa aceitação das massas sem glúten pelo público composto por indivíduos não celíacos, já que estão habituados com produtos à base de farinha de trigo.

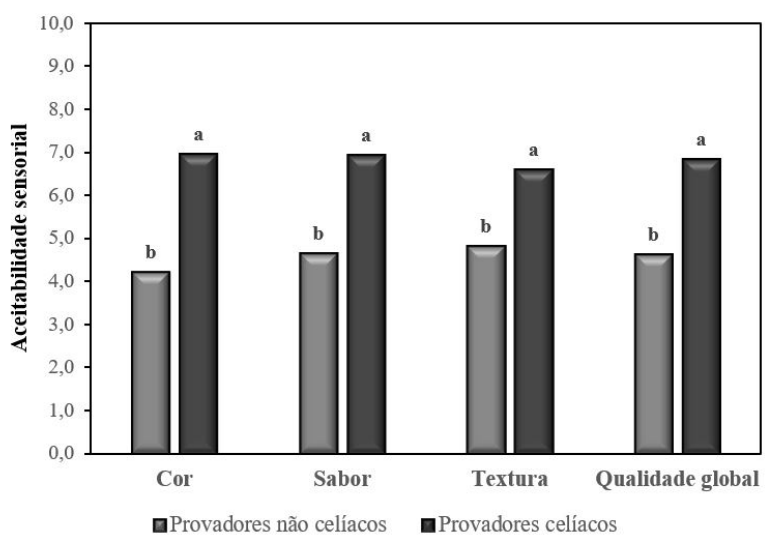

Figura 1. Aceitabilidade sensorial de macarrão à base de farinha de sorgo: comparação entre indivíduos celíacos (média de 39 repetições) e não celíacos (média de 74 repetições). Barras com letras diferentes são significativamente diferentes (Teste de Tukey, $p<0,05$ ).

Tabela 3. Resultados dos testes de aceitação com indivíduos não celíacos*.

\begin{tabular}{cccc}
\hline \multirow{2}{*}{ Atributos } & & Média & MM \\
\cline { 2 - 4 } & MS & MMS & $5,90^{\mathrm{a}}$ \\
\hline Cor & $4,20^{\mathrm{b}}$ & $6,01^{\mathrm{a}}$ & $7,11^{\mathrm{a}}$ \\
Sabor & $4,63^{\mathrm{b}}$ & $5,00^{\mathrm{b}}$ & $7,32^{\mathrm{a}}$ \\
\hline Textura & $4,80^{\mathrm{c}}$ & $5,76^{\mathrm{b}}$ & $6,82^{\mathrm{a}}$ \\
\hline
\end{tabular}

*Média de 74 repetições (indivíduos). Médias seguidas de letras diferentes na mesma linha são significativamente diferentes (Teste de Tukey, $p \leq$,05). MS: macarrão elaborado com $100 \%$ de farinha de sorgo; MSM: macarrão elaborado com $50 \%$ de farinha de sorgo e $50 \%$ de farinha de milho; MM: macarrão elaborado com $100 \%$ de farinha de milho.

Em trabalho semelhante, Giménez et al. (2015) avaliaram a aceitação de espaguete sem glúten à base de milho entre indivíduos celíacos e não celíacos. Da mesma forma, o painel de pessoas não celíacas não apreciou a massa sem glúten. No entanto, o produto foi bem aceito pelo público de indivíduos celíacos.

\section{Conclusões}

As massas de sorgo apresentaram alto valor nutricional, principalmente em relação à quantidade de proteínas e de fibras. Todas as massas sem glúten avaliadas neste trabalho apresentaram parâmetros de cozimento adequados. Os produtos MS e MSM precisam ser reformulados para obterem maior aceitação entre indivíduos não celíacos. No entanto, o público composto por indivíduos celíacos apreciou o macarrão de sorgo, o que indica uma alternativa de produto com qualidade nutricional e com possibilidade de custo menor que os produtos à base de outros cereais sem glúten, pelo fato de o sorgo ser um cereal com menor custo de produção no campo. 


\section{Agradecimentos}

Os autores agradecem à Empresa Brasileira de Pesquisa Agropecuária (Embrapa) e ao Conselho Nacional de Desenvolvimento Científico e Tecnológico $(\mathrm{CNPq})$, pelo suporte financeiro, e à Empresa TIVVA ${ }^{\circledR}$ (Sorocaba, São Paulo), pela fabricação das amostras.

\section{Referências}

AACC International Approved Methods - AACC. (2010). Approved Methods of Analysis. Method 16-50.02. Pasta and Noodle Cooking Quality-Firmness. St. Paul: AACC International.

Abuhammad, W., Elias, E. M., Manthey, F. A., Alamri, M. S., \& Mergoum, M. (2012). Comparison of methods for assessing dough and gluten strength of durum wheat and their relationship to pasta cooking quality. International Journal of Food Science \& Technology, 47(12), 2561-2573. http://dx.doi.org/10.1111/j.1365-2621.2012.03135.x

Alamprese, C., Casiraghi, E., \& Pagani, M. (2007). Development of gluten-free fresh egg pasta analogues containing buckwheat. European Food Research and Technology, 225(2), 205-213. http://dx.doi.org/10.1007/s00217-006-0405-y American Oil Chemists' Society - AOCS. (2004). Official methods and recommended practices of the American Oil Chemists Society. Method Am 5-04: Rapid determination of oil/fat utilizing high temperature solvent extraction. Champaign: AOCS.

ANKOM Technology. (2006). Neutral Detergent Fiber in Feeds - Filter Bag Technique (for A2000 and A2000I). NDF. Method 6. Retrieved in 2018, April 25, from https://www.ankom.com/embed-only/document/neutral-detergent-fiber-method-a2000

Anunciação, P. C., Cardoso, L. M., Gomes, J. V. P., Della Lucia, C. M., Carvalho, C. W. P., Galdeano, M. C., Queiroz, V. A. V., Alfenas, R. C. G., Martino, H. S. D., \& Pinheiro-Sant'Ana, H. M. (2017). Comparing sorghum and wheat whole grain breakfast cereals: sensorial acceptance and bioactive compound content. Food Chemistry, 221, 984-989. PMid:27979303. http://dx.doi.org/10.1016/j.foodchem.2016.11.065

Araújo, H. M. C., Araújo, W. M. C., Botelho, R. B. A., \& Zandonadi, R. P. (2010). Doença celíaca, hábitos e práticas alimentares e qualidade de vida. Revista de Nutrição, 23(03), 467-472. http://dx.doi.org/10.1590/S1415-52732010000300014

Arbex, P. M., Moreira, M. E. C., Toledo, R. C. L., Cardoso, L. M., Pinheiro-Sant'ana, H. M., Benjamin, L. A., Licursi, L., Carvalho, C. W. P., Queiroz, V. A. V., \& Martino, H. S. D. (2018). Extruded sorghum flour (Sorghum bicolor L.) modulate adiposity and inflammation in high fat diet-induced obese rats. Journal of Functional Foods, 42, 346-355. http://dx.doi.org/10.1016/j.jff.2018.01.010

Associação dos Celíacos do Brasil - ACELBRA. (2017). Quais produtos sem glúten você gostaria de encontrar com facilidade? Retrieved in 2018, December 7, from http://www.acelbra.org.br/2004/estatisticas.php

Association of Official Analytical Chemists - AOAC. (2016). Official Methods of Analysis of AOAC International (20th ed.). Rockville, Maryland: AOAC.

Awika, J. M., \& Rooney, L. W. (2004). Sorghum phytochemicals and their potential impact on human health. Phytochemistry, 65(9), 1199-1221. PMid:15184005. http://dx.doi.org/10.1016/j.phytochem.2004.04.001

Awika, J. M., McDonough, C. M., \& Rooney, L. W. (2005). Decorticating sorghum to concentrate healthy phytochemicals. Journal of Agricultural and Food Chemistry, 53(16), 6230-6234. PMid:16076098. http://dx.doi.org/10.1021/jf0510384

Awika, J. M., Rooney, L. W., \& Waniska, R. D. (2004). Anthocyanins from black sorghum and their antioxidant properties. Food Chemistry, 90(1-2), 293-301. http://dx.doi.org/10.1016/j.foodchem.2004.03.058

Biliaderis, C. G. (1992). Structures and phase transitions of starch in food systems. Food Technology, 46, 98-109.

Dicko, M. H., Gruppen, H., Traoré, A. S., Voragen, A. G. J., \& Van Berkel, W. J. H. (2006). Sorghum grain as human food in Africa: relevance of content of starch and amylase activities - review. African Journal of Biotechnology, 5(5), 384-395.

Dykes, L., Rooney, L. W., Waniska, R. D., \& Rooney, W. L. (2005). Phenolic compounds and antioxidant activity of sorghum grains of varying genotypes. Journal of Agricultural and Food Chemistry, 53(17), 6813-6818. PMid:16104804. http://dx.doi.org/10.1021/jf050419e

Ferreira, S. M. R., Luparelli, P. C., Schieferdecker, M. E., \& Vilela, R. M. (2009). Cookies sem glúten a partir da farinha de sorgo. Archivos Latinoamericanos de Nutricion, 59(4), 433-440. PMid:20677459.

Ferreira, S. M. R., Mello, A. P., Anjos, M. C. R., Krügera, C. C. H., Azoubeld, P. M., \& Alves, M. A. O. (2016). Utilization of sorghum, rice, corn flours with potato starch for the preparation of gluten-free pasta. Food Chemistry, 191, 147-151. PMid:26258714. http://dx.doi.org/10.1016/j.foodchem.2015.04.085

Giménez, M. A., Gámbaro, A., Miraballes, M., Roascio, A., Amarillo, M., Sammán, N., \& Lobo, M. (2015). Sensory evaluation and acceptability of gluten-free Andean corn spaghetti. Journal of the Science of Food and Agriculture, 95(1), 186-192. PMid:24752476. http://dx.doi.org/10.1002/jsfa.6704

Giuberti, G., Gallo, A., Cerioli, C., Fortunati, P., \& Masoero, F. (2015). Cooking quality and starch digestibility of gluten free pasta using new bean flour. Food Chemistry, 175, 43-49. PMid:25577049. http://dx.doi.org/10.1016/j.foodchem.2014.11.127

Güler, S., Köksel, H., \& Ng, P. K. W. (2002). Effects of industrial pasta drying temperatures on starch properties and pasta quality. Food Research International, 35(5), 421-427. http://dx.doi.org/10.1016/S0963-9969(01)00136-3

Hummel, C. (1966). Macaroni products: manufacture, processing and packing. Londres: Trade Press. 
Khan, I., Yousif, A. M., Johnson, S. K., \& Gamlath, S. (2015). Acute effect of sorghum flour-containing pasta on plasma total polyphenols, antioxidant capacity and oxidative stress markers in healthy subjects: a randomised controlled trial. Clinical Nutrition (Edinburgh, Lothian), 34(3), 415-421. PMid:25175757. http://dx.doi.org/10.1016/j.clnu.2014.08.005

Lucisano, M., Cappa, C., Fongaro, L., \& Mariotti, M. (2012). Characterisation of gluten-free pasta through conventional and innovative methods: Evaluation of the cooking behaviour. Journal of Cereal Science, 56(3), 667-675. http://dx.doi.org/10.1016/j.jcs.2012.08.014

Marengo, M., Bonomi, F., Marti, A., Pagani, M. A., Elkhalifa, A. E. O., \& lametti, S. (2015). Molecular features of fermented and sprouted sorghum flours relate to their suitability as components of enriched gluten-free pasta. Lebensmittel-Wissenschaft + Technologie, 63(1), 511-518. http://dx.doi.org/10.1016/j.Iwt.2015.03.070

Middleton Junior, E., Kandaswami, C., \& Theoharides, T. C. (2000). The effects of plant flavonoids on mammalian cells: implications for inflammation, heart disease, and cancer. Pharmacological Research, 52(4), 673-751. PMid:11121513.

Moraes, E. A., Natal, D. I. G., Queiroz, V. A. V., Schaffert, R. E., Cecon, P. R., De Paula, S. O., Benjamim, L. A., Ribeiro, S. M. R., \& Martino, H. S. D. (2012). Sorghum genotype may reduce low-grade inflammatory response end oxidative stress and maintains jejunum morphology of rats fed a hyperlipidic diet. Food Research International, 49(1), 553-559. http://dx.doi.org/10.1016/j.foodres.2012.07.029

Paiva, C. L., Queiroz, V. A. V., De Carvalho, C. W. P., \& Garcia, M. A. V. T. (2018). Acceptability and study of shelf life of gluten free cereal bar with popped and extruded sorghum based on a consumer acceptability. Caderno de Ciências Agrárias, 10, 5258.

Paiva, C. L., Queiroz, V. A. V., Simeone, M. L. F., Schaffert, R. E., Oliveira, A. C., \& Silva, C. S. (2017). Mineral contents in sorghum genotypes: influence of water stress. Food Chemistry, 214, 400-405. PMid:27507491. http://dx.doi.org/10.1016/j.foodchem.2016.07.067

Petitot, M., Boyer, L., Minier, C., \& Micard, V. (2010). Fortification of pasta with split pea and faba bean flours: pasta processing and quality evaluation. Food Research International, 43(2), 634-641. http://dx.doi.org/10.1016/j.foodres.2009.07.020

Phimolsiripol, Y., Mukprasirt, A., \& Schoenlechner, R. (2012). Quality improvement of rice-based gluten free bread using different dietary fibre fractions of rice bran. Journal of Cereal Science, 56(2), 389-395.

http://dx.doi.org/10.1016/j.jcs.2012.06.001

Queiroz, V. A. V., Aguiar, A. S., Menezes, C. B., Carvalho, C. W. P., Paiva, C. L., Fonseca, P. C., \& Conceição, R. R. P. (2018) A low calorie and nutritive sorghum powdered drink mix: Influence of tannin on the sensorial and functional properties. Journal of Cereal Science, 79, 43-49. http://dx.doi.org/10.1016/j.jcs.2017.10.001

Queiroz, V. A. V., Carneiro, H. L., Deliza, R., Rodrigues, J. A. S., Vasconcellos, J. H., Tardin, F. D., \& Queiroz, L. R. (2012). Genótipos de sorgo para produção de barra de cereais. Pesquisa Agropecuária Brasileira, 47(2), $287-293$. http://dx.doi.org/10.1590/S0100-204X2012000200018

Queiroz, V. A. V., Silva, C. S., Menezes, C. B., Schaffert, R. E., Guimarães, F. F. M., Guimarães, L. J. M., Guimarães, P. E. O., \& Tardin, F. D. (2015). Nutritional composition of [Sorghum bicolor (L.) Moench] genotypes cultivated without and with water stress. Journal of Cereal Science, 65, 103-111. http://dx.doi.org/10.1016/j.jcs.2015.06.018

Sęczyk, Ł., Świeca, M., \& Gawlik-Dziki, U. (2016). Effect of carob (Ceratonia siliqua L.) flour on the antioxidant potential, nutritional quality, and sensory characteristics of fortified durum wheat pasta. Food Chemistry, 194, 637-642. PMid:26471602. http://dx.doi.org/10.1016/j.foodchem.2015.08.086

Susanna, S., \& Prabhasankar, P. (2012). Quality, microstructure, biochemical and immunochemical characteristics of pypoallergenic pasta. Food Science \& Technology International, 18(4), 403-411. PMid:22859651. http://dx.doi.org/10.1177/1082013211428217

Teixeira, N. C., Queiroz, V. A. V., Rocha, M. A., Amorim, A. C. P., Soares, T. O., Monteiro, M. A. M., Menezes, C. B., Schaffert, R. E., Garcia, M. A. V. T., \& Junqueira, R. G. (2016). Resistant starch content among several sorghum (Sorghum bicolor) genotypes and the effect of heat treatment on resistant starch retention in two genotypes. Food Chemistry, 197(Pt A), $291-296$. PMid:26616952. http://dx.doi.org/10.1016/j.foodchem.2015.10.099

Tomicki, L., Rigo, A. A., Durigon, A., Gutkoski, L. C., Zeni, J., Valduga, E., Steffens, C., \& Toniazzo, G. (2015). Elaboration and quality evaluation of the gluten-free pasta. Ciência Rural, 45(7), 1311-1318. http://dx.doi.org/10.1590/0103-8478cr20140977

Villanueva, N. D. M., Petenate, A. J., \& Da Silva, M. A. A. P. (2005). Performance of the hybrid hedonic scale as compared to the traditional hedonic, self-adjusting and ranking scales. Food Quality and Preference, 16(8), 691-703.

http://dx.doi.org/10.1016/j.foodqual.2005.03.013

Wiles, P. G., Gray, I. K., \& Kissling, R. C. (1998). Routine analysis of protein by Kjeldahl and Dumas methods: review and interlaboratory study using dairy products. Journal of AOAC International, 81(3), 620-632. PMid:9606925.

Financiamento: Embrapa projeto SEG - 02.14.13.001.00.00. 